\title{
Carnets
}

Revue électronique d'études françaises de l'APEF

Deuxième série - 9 | 2017

Reconnaissances et légitimité en français

\section{La pluralité du moi dans quelques textes médiévaux}

Helder Godinho

\section{(2) OpenEdition}

Journals

Édition électronique

URL : http://journals.openedition.org/carnets/2035

DOI : $10.4000 /$ carnets.2035

ISSN : 1646-7698

\section{Éditeur}

APEF

\section{Référence électronique}

Helder Godinho, «La pluralité du moi dans quelques textes médiévaux », Carnets [En ligne], Deuxième série - 9 | 2017, mis en ligne le 31 janvier 2017, consulté le 01 mai 2019. URL : http:// journals.openedition.org/carnets/2035; DOI : 10.4000/carnets.2035

Ce document a été généré automatiquement le 1 mai 2019.

\section{(c) (i) (8)}

Carnets est mis à disposition selon les termes de la licence Creative Commons - Atribution - Pas d'utilisation commerciale 4.0 International. 


\title{
La pluralité du moi dans quelques textes médiévaux
}

\author{
Helder Godinho
}

1 Je me réjouis de participer à ce numéro de la revue Carnets, non seulement parce que ma vie est très liée aux études françaises mais aussi parce que l'époque de la littérature française que j'ai le plus étudiée, le Moyen Âge, a connu un rayonnement spécial en France, surtout aux $\mathrm{XII}^{\mathrm{e}}$ et $\mathrm{XIII}^{\mathrm{e}}$ siècles, siècles dont les thématiques et les formes ont marqué tout le Moyen Âge européen : le roman, le cycle du Graal et toute la littérature arthurienne avec, entre autres, Tristan. Et c'est justement de ce Moyen Âge que je vais partir pour vous parler ici d'un sujet qui m'intéresse particulièrement : la complexité du moi, à laquelle le Moyen Âge s'est beaucoup intéressé, et les formes qu'elle a prises à cette époque et qui dépendaient des outils conceptuels disponibles.

2 En effet, la littérature médiévale en général, et pas seulement celle des $\mathrm{XII}^{\mathrm{e}}$ et $\mathrm{XIII}^{\mathrm{e}}$ siècles, a beaucoup réfléchi sur la complexité du moi, surtout autour de la figure du héros, utilisant pour cela une thématique traditionnelle véhiculée aussi par des hagiographies et des textes sacrés, comme la Bible. Cette thématique dépasse en amont et en aval le Moyen Âge et se prolonge jusqu'à nos jours sous des formes diverses où elle est soutenue par tout un raisonnement philosophique et psychologique, comme on sait, avec les outils conceptuels de notre époque. Au Moyen Âge fondamentalement chrétien, la figure du Christ lui-même, avec ses deux natures, humaine et divine, favorisait, naturellement, cette réflexion. C'est sa nature divine qui a conduit sa vie et qui a déterminé tout son parcours, comme Jésus le rappelle à sa mère quand elle l'a cherché dans le Temple où il parlait avec les Docteurs.

3 Or, le Moyen Âge a multiplié les occurrences de cette double nature, humaine et divine, à partir des vies de saints. Comme exemples, prenons les vies de saint Alexis (Perugi, 2000) et de sainte Euphrosyne (« Vidas de Santos... », vol. II, 1985).

Ces saints, comme beaucoup d'autres, sont des enfants de couples stériles où l'intervention directe de Dieu a été demandée par ses parents humains. D'où il résulte que les enfants ainsi conçus sentent un appel à une vie au-delà de l'humanité : Alexis laisse la femme avec qui il venait de se marier et part pour mener une vie qui le conduira à la 
sainteté ; Euphrosyne, dont l'intervention de Dieu qui a permis sa naissance a été demandée par ses parents humains et aussi par l'abbé d'un couvent, fuit le mariage humain que son père veut lui imposer et, pour ne pas être recherchée dans les couvents de femmes, va, en se déguisant en homme, se cacher et vivre jusqu'à la fin de ses jours dans le couvent d'hommes de l'abbé qui avait prié aussi pour sa naissance. Leurs destinées exceptionnelles sont ainsi basées sur une sorte de double nature, du fait de l'intervention directe de Dieu pour leurs naissances.

Beaucoup de héros, aussi, surtout les plus importants à l'échelle de la culture et de l'histoire, ont une double nature en étant fils d'un dieu et d'une mortelle, comme Héraclès, ou d'une déesse et d'un mortel, comme Énée. Cette double nature "s'affaiblit ", pourrait-on dire, dans les cas, très nombreux, de double parentalité humaine : je ne cite, comme exemple, que Moïse, adopté par la fille du Pharaon, ou đEdipe, élevé par un berger. Cette problématique «foisonne» au Moyen Âge, non seulement pour les êtres exceptionnels, héros ou saints, mais aussi pour le commun des mortels. L'importance du folklore, spécialement le fonds celte, a beaucoup contribué à cela. Prenons quelques exemples.

6 Le père du roi Arthur, Utherpendragon, s'appelait simplement Uther jusqu'au moment où son frère Aurelius Ambrosius (ou Pendragon) meurt (Boron, 1979: 122) et il devient roi : il s'appellera, alors, Utherpendragon en mémoire d'une étoile qui semblait un dragon et qui était apparue au moment de la mort de son frère. Il est bien évident que ce nouveau nom est le signe d'une incorporation d'un autre qui renforce son pouvoir et sa richesse puisqu'il a hérité de sa terre, en devenant roi. Quelque temps après, en voulant coucher avec la duchesse Ygerne, il prend, grâce à Merlin, l'aspect du mari de celle-ci, et c'est dans cette condition de l'autre duquel il a pris la figure qu'il engendre Arthur au château de Tintagel (Monmouth, 1958). Arthur, qui aura un avenir glorieux, est donc le fils d'un homme qui cumule trois personnages (le sien, celui de son frère et celui du duc mari d'Ygerne), en créant par-là un moi vraiment pluriel mais sans perdre son identité (son ipséité, pourrait-on dire). Et, puisque Merlin a collaboré à la naissance d'Arthur, souvenons-nous tout de suite qu'il est le fils d'une mortelle et d'un incube, par lequel il a la connaissance du Passé, connaissance à laquelle Dieu ajoutera la connaissance du Futur (Boron, 1979 : 49-50).

7 Arthur est donc le fruit de personnes très complexes qui se composent de plusieurs personnages. De la sorte, il intègre toute cette complexité ce qui lui donne une nature apte aux grands faits, comme les héros antiques dont on a parlé, parce que cette complexité permet en quelque sorte un choix entre les natures, quoique la grandeur de la nature non-humaine la rende trop désirable pour ne pas être choisie. Alexis et Euphrosyne choisissent leur nature «divine ", donnée par l'intervention directe de Dieu dans leur naissance, et c'est pourquoi ils n'obéissent pas à leurs pères humains. Ce choix est le fruit de ce qu'on pourrait appeler une prédestination qui consiste à donner aux prédestinés la possibilité de choisir une nature plus qu'humaine, choix qui ne peut pas faire abstraction de la supériorité de la seconde nature qui oblige à la grandeur, au refus de la banalité d'une vie commune. Ce qui veut dire que la complexité du moi est le socle d'une liberté basée sur la possibilité de choisir, même si l'attrait de la seconde nature rend presque inévitable la direction du choix. Dans ces contextes, la grandeur veut dire création d'empires ou de qualités divines dans l'humain (la sainteté mais aussi l'héroïsme des héros avec un parent divin) et refus de la simple humanité enfermée dans la banalité noncréatrice. 
8 Si l'on revient à Arthur, l'épisode de l'épée enfoncée dans la pierre et que lui seul peut retirer montre une prédestination, c'est-à-dire une préséance de l'autre nature ou des autres natures qui le met au-dessus de la nature purement humaine (Boron, 1979: 268-275). Et cette préséance qui oriente le choix de façon presque obligatoire (la prédestination) est possible parce que ces possibilités diverses sont inscrites dans le moi qui choisit en tant que composé de plusieurs natures. Quoique la prédestination et le choix semblent contradictoires il s'agit ici d'un choix du meilleur et du plus grand auquel ces autres natures donnent accès. Le choix est possible parce que le moi a d'autres natures inscrites en lui qui ouvrent la vie à des possibilités non-banales.

Le thème traditionnel de la double paternité ou de la double maternité (Héraclès, par exemple) est une des premières affirmations de la complexité du moi et c'est parce qu'il est complexe et pluriel qu'il peut choisir la grandeur au-delà des faits divers et des choix du quotidien banal. Et ce thème, dont le Christ est un des exemples, se prolonge à travers les siècles ; la littérature traditionnelle, entre autres sources, l'atteste, comme j'ai essayé de montrer dans une autre étude (Godinho, 2010 : 265-276).

10 La littérature médiévale nous parle aussi d'une complexité intrinsèque à la nature humaine elle-même, où le moi abrite plusieurs personnages. Prenons, par exemple, le roman du XIII siècle, Gui de Warewic (Ewert, 1932-33). Gui, le fils du sénéchal du comte de Warwick, tombe amoureux de la fille du comte qui lui demande, pour suppléer à sa condition sociale inférieure, de devenir le meilleur chevalier du monde pour qu'elle lui accorde son amour. C'est donc à un personnage plus qu'à un homme qu'elle veut avoir affaire et c'est à ce personnage qu'elle se mariera après qu'il est finalement devenu le meilleur chevalier du monde. Cinquante jours après ce mariage, avec sa femme enceinte, Gui monte à une tour, voit son domaine et son bonheur mais pense qu'il n'a jamais rien fait pour son Créateur, étant donné les crimes qu'il avait commis pour devenir le meilleur chevalier du monde. Il décide alors de quitter sa femme et de devenir ermite, en donnant par-là corps à un autre personnage que sa femme n'a même pas le droit de visiter et, quand elle meurt, quelques années plus tard, elle ne peut même pas être enterrée à côté de lui. Déjà donnant corps à ce personnage de l'ermite repentant, il rencontre un ancien ami qui, sans le reconnaître, lui dit qu'il cherche le fils de Gui (son fils, donc) que les arabes ont enlevé. Gui s'en va sans s'intéresser au sort de son fils, comme si la coupure entre le personnage «meilleur chevalier du monde », laquelle avait généré ce fils, et celui d' «ermite » était radicale : il est maintenant une sorte d'autre différent du meilleur chevalier du monde qui avait généré ce fils.

11 Nous voyons ainsi qu'il a pu choisir un nouveau chemin parce qu'il pouvait donner corps à plusieurs personnages, sortis, évidemment, de sa complexité intérieure. Il choisit de passer du statut de héros à celui de saint, cette fois non parce qu'il a une autre nature donnée par une parentalité plus qu'humaine, mais parce que son moi peut, lui-même, se doubler pour changer son rapport à la vie, il n'a pas une substantivité fermée. Et cela lui permet de choisir, c'est-à-dire d'être libre.

12 Utherpendragon, le père d'Arthur, avait un moi composé des personnages qu'il avait intégrés, son frère et le mari d'Ygerne, dont il prend la forme pour générer Arthur. Avec ceci, on entre dans le très intéressant domaine des métamorphoses. Merlin, qui avait "métamorphosé » Utherpendragon pour qu'il prenne la forme de l'autre qu'il veut remplacer, se métamorphose lui-même. Le fonds culturel qui est à l'origine de ce genre de personnages et de phénomènes n'est pas notre objet d'étude aujourd'hui. Ce que je veux c'est remarquer que la persistance de ce genre de phénomènes dans la littérature et le 
folklore montre qu'il est la surface d'un fonds imaginaire qui m'intéresse ici. Il me semble qu'il est une des "variantes " possibles de la complexité d'un moi qui s'affirme comme une tension entre des possibilités internes diverses ce qui l'oblige à se mouvoir dans la recherche d'un manque, d'une absence (ou d'un signifiant absent).

Dans ce sens vont aussi les Fées (Harf-Lancner, 1984). Marraines, elles décident des destinées humaines, comme les Parques antiques et font que ce qui meut la vie d'un humain ne dépends pas que de lui. Cette vie est une tension entre «lui » et l'autre que la Fée l'oblige d'être en ayant déterminé une destinée qu'il n'a pas choisie. Amantes, elles ouvrent aux humains leur monde non-humain et, dans le cas où elles donnent des enfants aux humains comme Mélusine et les Fées "mélusiniennes ", génèrent même des êtres à double nature (humaine et faée). C'est-à-dire que ce thème traditionnel déborde la littérature et le folklore car il s'insère dans toute une tradition héroïque, hagiographique et même simplement humaine qui parle, avec des outils divers de ceux de la pensée d'aujourd'hui, de la complexité plurielle du moi, de la recherche obligatoire d'un autre qui nous habite et qui s'affirme comme une absence présente et incontournable. Ceux qui ont marqué, comme héros ou saints, le devenir humain, sont explicitement présentés comme possédant une nature au moins double (Merlin semble avoir une triple nature, le père d'Arthur aussi, entre autres) qui change l'ordre humain des faits et des valeurs parce que, pour orienter sa vie, elle choisit son côté divin (ou, simplement, plus qu'humain, comme ceux qui ont une destinée faée).

14 La culture est, ainsi, un mouvement vers la connaissance et la création ou transformation des valeurs mû par l'autre inscrit dans l'humain, puisque c'est lui qui est responsable des hauts faits, ce que l'exceptionnalité des héros et des saints dit explicitement. Dans l'Occident chrétien, cela se valorise sur le fonds d'un Dieu trinitaire (mais la complexité divine n'est pas exclusive de l'Occident, comme on sait). La pensée contemporaine, dans son questionnement du Sujet, reconnaît l'importance de ces thèmes traditionnels, reconnaissance qui relie la littérature et la pensée moderne dans plusieurs de ses disciplines.

15 Cette complexité du moi a été un sujet toujours si important, qu'elle est dite sous d'autres formes encore, qui peuvent sembler plus lointaines. Si l'on reprend la littérature médiévale, on remarque un traitement du thème de l'ombre, un sujet universel de grande importance, comme on sait, qui y fonctionne comme un thème complexe et prégnant. Par exemple, Jean Renart, auteur français du XIII ${ }^{\mathrm{e}}$ siècle, non seulement introduit, dans le Lai de l'Ombre, une réflexion très intéressante sur l'ombre, mais réfléchit aussi, dans le Roman de la Rose ou de Guillaume de Dôle, sur la narrativité du moi (Renart, 1979). Dans le Lai de l'Ombre, un chevalier, qui est amoureux d'une Dame qui refuse son amour et l'anneau qu'il lui veut mettre au doigt, remarque l'ombre de la Dame reflétée dans l'eau du puits auprès duquel ils sont et a l'inspiration de jeter l'anneau sur l'ombre de la Dame. En conséquence, elle se met à l'aimer tout de suite (Renart, 1997 : 578-631). Et Jean Renart conclut qu'il a fait preuve de grande intelligence. En effet, cette ombre était une image de l'autre de la Dame, cette ombre était, selon le chevalier, l'amie que la Dame refusait d'être, et dont l'amour de la Dame avait besoin pour se mettre à exister.

Passons au Guillaume de Dôle. Là, l'empereur Konrad, devenu amoureux d'une jeune fille par ouï-dire, au lieu d'aller la connaître personnellement ou de la faire venir à la Cour, fait venir à la Cour le frère de la fille pour qu'il raconte des histoires sur elle qui montrent qui elle est. C'est-à-dire que ce qui comptait pour qu'elle soit aimée n'était pas elle dans l'unité de son corps mais ce qu'elle signifiait et qui était montré dans l' «ombre » d'elle 
que les histoires construisaient. Ce que l'autre vaut pour être aimé c'est ce qu'il signifie et cette signification est une sorte d'ombre dans laquelle l'amour peut prendre vie, comme dans le Lai de l'Ombre. Cette ombre est bien le lieu où la relation peut se faire ou se défaire, au cas où l'ombre et la signification qu'elle véhicule changent. La valeur de l'autre comme être aimé dépend de son rôle en tant que personnage du récit qu'on raconte sur lui. Ce qui est dit clairement dans un autre roman du XIII ${ }^{\mathrm{e}}$ siècle, Durmart le Gallois (Gildea, 1965), où la femme aimée par ouï-dire, dans un récit où elle avait pris signification, n'est aimable qu'en tant que personnage de ce récit - et c'est pourquoi quand Durmart la rencontre sans savoir qui elle est (donc à un moment où elle n'est pas le personnage aimé du récit) il ne sent rien pour elle et ne se met à l'aimer que quand la même femme est reconnue comme étant le personnage du récit qu'il cherchait, c'est-à-dire la reine d'Irlande.

L'amour de loin, l'amour par ouï-dire, est normalement traité d'une façon distraite par la critique. Pourtant, saint Augustin, quelques siècles auparavant, avait déjà reconnu son importance. Dans les Confessions, il remarque, à propos du rhéteur Hiérius, à qui il dédie même un livre sans le connaître, que l'amour qu'il sentait pour lui venait de ce qu'il avait entendu raconter de lui et qui était positif, les gens lui reconnaissant grande valeur. Et il remarque que si Hiérius avait fait les mêmes choses et qu'on disait de lui la même chose, mais d'une façon négative, avec un jugement négatif, il ne l'aurait pas aimé (Santo Agostinho, 2000, 151-153). C'est-à-dire, pour revenir au contexte de Jean Renard, l'autre est aimable dans son ombre, dans ce qu'il signifie pour celui qui va l'aimer, et cela est dit et induit par celui qui parle de lui, comme le frère de Léonor, la fille que l'empereur aimait. Celui qui construit le récit est l'autre extérieur qui va créer la signification. L'autre est donc aimable quand il est doublé deux fois : par les mots qui lui assignent une signification et par l' " ombre » qu'elle produit. Ce qui veut dire, il me semble, qu'un même processus est à l'œuvre pour faire un héros, un saint ou un amant : la complexité plurielle du moi crée par double nature héritée ou crée par un dédoublement de l'autre dans le sens qu'il prend pour celui qui l'aime et que l'on peut trouver dans l'image renardienne de l'ombre. Aux héros ou aux saints à qui un parent divin avait donné une autre nature, font pendant les simples humains qui sont devenus aimables parce que quelqu'un a créé, comme un parent divin, dans ce qu'il raconte, un sens équivalent de l'autre nature divine des héros ou des saints, sens que l'ombre renardienne signifie.

N'oublions pas aussi, et ceci est plein de conséquences, que l'amour envers Dieu est un amour de loin par oui-dire créé par un récit doctrinal ou mythique. Pour aimer le vrai Dieu il faut avoir le vrai récit que les Fondateurs ont fait de la même façon que ceux qui évoquaient Hiérius ou les vertus qui faisaient aimer la femme médiévale. Il faut donc aussi un tiers pour que cette relation soit possible. Le choix dépend de l'autre qui raconte et valorise, comme pour les héros et les saints le choix dépendait de l'autre nature inscrite en lui. La complexité du moi dans les diverses formes qu'elle prend, internes (l'autre comme nature-plus-que-humaine) ou externes (l'autre comme personnage ou comme ombre significative crée par un récit externe qui nous lie à l'autre en nous donnant un sens pour l'aimer, même si l'autre est Dieu) est un thème qui prend des formes diverses dans la littérature et la culture et qu'il serait bon de ne pas méconnaître dans la réflexion actuelle qui reconnaît cette complexité avec des outils de pensée différents.

Autrefois on se masquait pour les voyages chamaniques et, comme maintenant encore, pour les Carnavals qui préparent l'accès au Chaos primordial (Caillois, 2008: 263-289) pour refaire un nouveau Cosmos (un nouvel an). L'accès à l'Autre Monde est fait par un 
autre que nous, comme le montre le besoin de se masquer. L'accès à Dieu et à son amour est fait grâce au texte qui le définit comme objet d'amour (ou de crainte) créé par les Fondateurs. L'accès à l'autre aimé dans le récit qui le raconte a besoin du récit et de son créateur (son auteur) ${ }^{1}$. La littérature, le folklore et les mythes ne font que parler, avec les outils conceptuels dont ils disposent, d'une pluralité du moi internalisé dans les doubles natures ou externalisé dans la médiation obligatoire pour l'autre (qui peut être signifiée par l'ombre) et pour Dieu. D'ailleurs, avec des outils conceptuels différents, la deuxième topique de Freud montre le Moi comme un lieu de rencontre du Ça et du Surmoi. La moderne notion d'inconscient a besoin de cette pluralité que les hommes ont toujours mise en scène, et ils l'ont fait par des artifices littéraires ou mythiques.

Les mythes et les littératures des époques passées, dont la période médiévale, ont fait de cette notion d'un moi pluriel et complexe un des thèmes les plus récurrents et importants, exprimé d'une façon diverse, pourrait-on dire, à travers des termes et des images plus ou moins clairs, parfois peu clairs pour un regard distrait. Aujourd'hui, les nouveaux outils intellectuels et la connaissance comparative des littératures, des mythes et des traditions montrent cette pluralité à l'œuvre presque partout, de façons parfois moins claires et rusées. Les Sciences Sociales et Humaines d'aujourd'hui l'ont reconnu et le traitent avec d'autres outils, mais ce qui est intéressant, c'est de retrouver un même sujet de réflexion à travers les âges et les formes qu'il a prises en accord avec les outils conceptuels disponibles.

\section{BIBLIOGRAPHIE}

BORON, Robert de (1979). Merlin. Roman du XIII siècle. Genève : Droz.

CAILloIS, Roger (2008 [1939]). «Le sacré de transgression : théorie de la fête » in Euvres. Paris : Gallimard Quarto.

EWERT, Alfred (1932-1933). Gui de Warewic. Roman du XIII siècle. Paris : Champion.

GodinHo, Helder (2010). « A Dupla Natureza em alguns Contos tradicionais », in Memórias, Gestos, Palavras. Textos oferecidos a Teresa Rita Lopes. Lisboa : Assírio e Alvim.

HARF-LANCNER, L. (1984). Les Fées au Moyen Age. Paris : Champion.

GILDEA, Joseph (1965). Durmart le Galois. Pennsylvania: The Villanova Press.

PERUGI, Maurizio (2000). La vie de saint Alexis (2000). Genève: Droz.

MONMOUTH, Geoffrey of (1958). History of the Kings of Britain [Historia Regum Britanniae]. New York: E. P. Dutton. Voir Book VI, chap. 18: 133-134; Book VIII, chap. 15: 170, chap. 17 et 19: 171-177.

RENART, Jean (1979). Le Roman de la Rose ou de Guillaume de Dole. Paris : Champion.

RENART, Jean (1997). « Le Lai de l'Ombre », in Suzanne Méjean-Thiolier et Marie-Françoise NotzGrob (éd.). Nouvelles Courtoises. Paris : Poche, Lettres Gothiques.

SANTO AGOSTINHo (2000). Confissões. Lisboa : INCM. 
Vidas de Santos de Um Manuscrito Alcobacense (Colecção Mística de Fr. Hilário da Lourinhã, COD. ALC. CCLXVI/ANTT 2274) (1985). Lisboa : Centro de Estudos Geográficos [separata].

\section{NOTES}

1. Il faudrait réfléchir, dans cet ordre d'idées, sur ce qu'est « l'auteur ».

\section{RÉSUMÉS}

Dans les textes et les personnalités ici mentionnées, nous rencontrons des personnages multiples (qui réunissent plusieurs identités) quand il s'agit de personnages qui ont eu un grand rôle dans l'histoire (réelle ou supposée) : créateurs d'empires, héros, saints, par exemple. Ils ont une nature double humaine et divine, comme Énée ou Héraclès, ou même les saints pour la naissance desquels Dieu a intervenu directement, ou une nature simplement humaine mais qui réunit plusieurs actants, comme Utherpendragon, le père du roi Arthur. Cela est interprété comme étant une forme de parler de la complexité du moi avec des outils conceptuels de l'époque.

The texts and the characters hear referred to, show us characters who have a double identity when it comes to persons with an important role in history: empire makers, heroes, saints, for example. They have a double nature, human and divine, as Aeneas or Heracles, or even the saints created by a direct intervention of God. Others have a nature only human but which is formed by several actors, as Utherpendragon, King Arthur's father. These facts are interpreted as being a way of referring to the complexity of the ego with the conceptual utensils of the epoch.

\section{INDEX}

Mots-clés : Moyen Âge, identité, complexité du moi, héros, Dieu

Keywords : Middle Age, identity, complexity of the ego, hero, God

\section{AUTEUR}

\section{HELDER GODINHO}

Un. Nova de Lisboa

ghpl[at]fcsh.unl.pt 